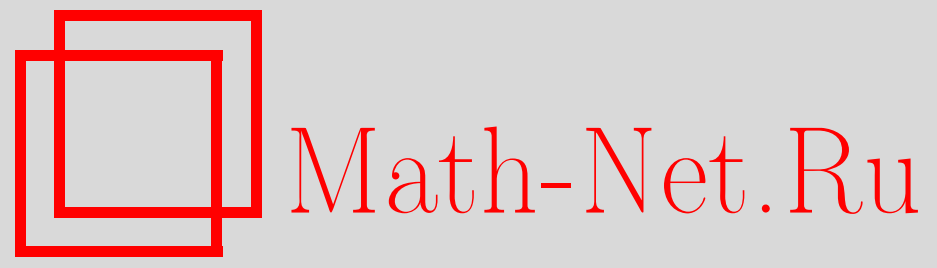

А. Н. Дёгтев, Д. И. Иванов, Слабо импликативно селекторные множества размерности 3, Дискрет. матем., 1999, том 11, выпуск 3, 126-132

DOI: https://doi.org/10.4213/dm379

Использование Общероссийского математического портала Math-Net.Ru подразумевает, что вы прочитали и согласны с пользовательским соглашением http://www.mathnet.ru/rus/agreement

Параметры загрузки:

IP : 3.91 .87 .62

26 апреля 2023 г., 03:32:35 


\title{
Слабо импликативно селекторные множества размерности 3
}

\author{
(c) 1999 г. А. Н. Дегтев, Д. И. Иванов
}

\begin{abstract}
По $n$-местной булевой функции $\beta$ определяется класс $K(\beta)$ слабо $\beta$-импликативно селекторных множеств, являющихся подмножествами натуральных чисел. Размерность класса $K(\beta)$ - это число существенных переменных в $\beta$. Описаны, с точностью до включения, все классы $K(\beta)$ размерностей 2 и 3 , за исключением одного случая.
\end{abstract}

Данная работа в идейном плане является продолжением статьи [1] и близка по духу работам $[2,3]$. Пусть $A \subseteq \mathrm{N}=\{0,1,2, \ldots\}$ и $\beta$ - $n$-местная булева функция (БФ) без фиктивных переменных. Если $x \in \mathrm{N}$, то положим $\bar{x}=1$, если $x \in A$, и $\bar{x}=0$ в противном случае. Назовем $A$ слабо $\beta$-импликативно селекторным $(\beta$-ИС) множеством, если существует $n$-местная частично-рекурсивная функция (ЧРФ) $f$ такая, что

$$
\left(\forall x_{1}, \ldots, x_{n}\right)\left(\beta\left(\bar{x}_{1}, \ldots, \bar{x}_{n}\right)=1 \Rightarrow f\left(x_{1}, \ldots, x_{n}\right) \in A \cap\left\{x_{1}, \ldots, x_{n}\right\}\right) .
$$

Говорим также, что $f$ соответствует $A$. Положим

$$
K(\beta)=\{A \subseteq \mathbf{N}: A-\beta \text {-ИС множество }\},
$$

а число $n$ назовем размерностью класса $K(\beta)$.

Считаем БФ $\beta$ допустимой, если $\beta(0, \ldots, 0)=0$. Ясно, что если $\beta$ не является такой, то $K(\beta)=\{\mathbf{N}\}$ и этот случай неинтересен. Поэтому ниже под БФ понимаем только допустимые булевы функции.

Лемма 1. Для любой БФ $\beta$

$$
K(x \vee y) \subseteq K(\beta)
$$

Доказательство. Пусть $\beta$ - $n$-местная БФ, $x_{1}, \ldots, x_{n} \in \mathrm{N}$, а $A$ - слабо $x \vee y$ ИС множество с соответствующей ЧРФ $f(x, y)$. Можно считать, что $f(x, x)=x$ и $f(x, y)=f(y, x)$. Ищем $a \in\left\{x_{1}, \ldots, x_{n}\right\}$ такое, что $f\left(a, x_{i}\right) \downarrow$, то есть определено для всех $i, 1 \leqslant i \leqslant n$. Если такого $a$ нет, то полагаем $g\left(x_{1}, \ldots, x_{n}\right) \uparrow$, то есть неопределено. Если $f\left(a, x_{i}\right)=a$ для всех $i$, то полагаем $g\left(x_{1}, \ldots, x_{n}\right)=a$. Иначе, пусть $y_{1}, \ldots, y_{m} \in$ $\left\{x_{1}, \ldots, x_{n}\right\}$ такие, что $f\left(a, y_{j}\right)=y_{j}$ и $y_{j} \neq a$ для всех $j, 1 \leqslant j \leqslant m$. Ясно, что $m<n$ и если $\left\{x_{1}, \ldots, x_{n}\right\} \cap A \neq \varnothing$, то и $\left\{y_{1}, \ldots, y_{m}\right\} \cap A \neq \varnothing$. Теперь продолжаем такую процедуру дальше, заменяя $x_{1}, \ldots, x_{n}$ на $y_{1}, \ldots, y_{m}$. Если $\left\{y_{1}, \ldots, y_{m}\right\} \cap A \neq \varnothing$, то через конечное число шагов обладающий указанными выше свойствами элемент $a$ будет найден. Тогда значение $g\left(x_{1}, \ldots, x_{n}\right)$ будет определено и принадлежать $A$. Поэтому $A \in K(\beta)$, и лемма 1 доказана. 
Так как любое рекурсивно-перечислимое множество (РПМ) является слабо $x \vee y$ ИС, то все они лежат в каждом из $K(\beta)$.

Лемма 2. Если ү получена из БФ $\beta$ в резулътате отождествления некоторых переменных или подстановки вместо некоторых переменных константы 0 , то $K(\beta) \subseteq K(\gamma)$

Доказательство. Смотрите лемму 2 из [1].

Лемма 3. Если $\beta \rightarrow \gamma-$ тождественная истинная БФ, то

$$
K(\gamma) \subseteq K(\beta)
$$

Доказательство. Если $A$ является слабо $\gamma$-ИС множеством с ЧРФ $f$, то $A$ является и слабо $\beta$-ИС множеством с этой же ЧРФ.

Лемма 4. Если $\beta\left(\bar{x}_{1}, \ldots, \bar{x}_{n}\right) \leqslant \bar{x}_{i} \partial л я$ подходящего $i \leqslant n$, то

$$
K(\beta)=\{A: A \subseteq N\} .
$$

Доказательство. Если $\beta\left(\bar{x}_{1}, \ldots, \bar{x}_{n}\right)=1$, то $\bar{x}_{i}=1$. Поэтому любое $A \subseteq N$ оказывается слабо $\beta$-ИС множеством с соответствующей общерекурсивной функцией $f\left(x_{1}, \ldots, x_{n}\right)=x_{i}$.

Следствие 1. Среди всех классов $K(\beta)$ класс $K(x \vee y)$ является наименъшим, $а$ $K(x y)$ наиболъиим.

Разумеется, ниже будут рассматриваться БФ с точностью до перестановки переменных. Классов $K(\beta)$ размерности 2 всего четыре:

$$
K(x \vee y), \quad K(x \oplus y)=K(x \bar{y} \vee \bar{x} y), \quad K(x \bar{y}), \quad K(x y)
$$

По лемме $4 K(x \bar{y})=K(x y)$. По следствию 1

$$
K(x \vee y) \subset K(x \oplus y) \subset K(x y)
$$

причем, как будет видно ниже, включения везде строгие. Авторами, с использованием определенной техники, были выписаны все (допустимые) БФ от трех переменных. Их (с точностью до перестановок переменных) оказалось 34.

Из леммы 4 вытекает следующее утверждение.

Лемма 5. Справедливо равенство

$$
K(\beta)=K(x y)
$$

если $\beta$ - одна из следующих 8 БФ:

$$
x y z, \quad x(y \vee z), \quad x(y \vee \bar{z}), \quad x(y z \vee \overline{y z}), \quad x y \bar{z}, \quad x \overline{y z}, \quad x(\bar{y} \vee \bar{z}), \quad x(y \bar{z} \vee \bar{y} z)
$$

Положив $z=0$, из леммы 2 получаем следующее утверждение. 
Лемма 6. Справедливо равенство

$$
K(\beta)=K(x \vee y),
$$

если $\beta$ - одна из следующих 11 БФ:

$x \vee y \vee z, \quad x \vee y \bar{z}, \quad x \vee y \bar{z} \vee \bar{y} z, \quad x \bar{y} \vee x \bar{z} \vee \bar{x} y, \quad x y \vee \bar{z}(x \vee y) \vee \overline{x y} z$, $x y \vee x \bar{z} \vee y \bar{z}, \quad x \bar{z} \vee \bar{x} y, \quad \bar{z}(x \vee y), \quad \bar{x} y \vee x \bar{z} \vee \bar{y} z, \quad x \bar{z} \vee y \bar{z} \vee \overline{x y} z, \quad x \bar{z} \vee y \bar{z} \vee \bar{y} z$.

Отождествив $z$ с $y$, из леммы 2 получаем следующее утверждение.

Лемма 7. Справедливо равенство

$$
K(\beta) \doteq K(x \vee y),
$$

если $\beta$ - одна из следующих 5 БФ:

$$
x \vee y z, \quad x \bar{z} \vee y z, \quad y z \vee x \overline{y z}, \quad x y \vee x \bar{z} \vee \bar{x} z, \quad y z \vee y \bar{z} \vee x \overline{y z}
$$

Осталось рассмотреть следующие 10 БФ, к которым добавлены еще $x y, x \vee y$ и $x \oplus y=x \bar{y} \vee \bar{x} y:$

$$
\begin{aligned}
\beta_{0} & =x \vee y, \\
\beta_{1} & =x \oplus y \oplus z=x y z \vee x y \bar{z} \vee x \bar{y} z \vee \bar{x} y z, \\
\beta_{2} & =x y \vee x z \vee y z, \\
\beta_{3} & =x \oplus y \\
\beta_{4} & =x y \bar{z} \vee x \bar{y} z \vee \bar{x} y z, \\
\beta_{5} & =x y \\
\beta_{6} & =x \bar{y} \vee x \bar{z} \vee \bar{x} y z \\
\beta_{7} & =x y z \vee x \overline{y z} \vee \bar{x} y \bar{z}, \\
\beta_{8} & =x \overline{y z} \vee \bar{x} y \bar{z} \vee \bar{x} y, \\
\beta_{9} & =\bar{z}(x \bar{y} \vee \bar{x} y) \\
\beta_{10} & =x \bar{y} \vee \bar{x} y z \\
\beta_{11} & =x \bar{y} \vee \bar{x} y \bar{z} \\
\beta_{12} & =x \overline{y z} \vee \bar{x} y \bar{z} .
\end{aligned}
$$

Ниже вместо $K\left(\beta_{i}\right), 0 \leqslant i \leqslant 12$, пишем $K_{i}$.

Лемма 8. Справедливы равенства

$$
K_{3}=K_{9}=K_{10}=K_{11}=K_{12} .
$$

Доказательство. Так как $\beta_{i} \rightarrow \beta_{3}$ при $9 \leqslant i \leqslant 12$ суть тождественно истинные БФ, по лемме $3 K_{3} \subseteq K_{i}$. Но

$$
\beta_{9}(x, y, 0)=\beta_{10}(x, y, y)=\beta_{11}(x, y, 0)=\beta_{12}(x, y, y)=\beta_{3}(x, y) .
$$

По лемме $3 K_{i} \subseteq K_{3}$, и лемма 8 доказана. 
Лемма 9. Справедливо равенство

$$
K_{3}=K_{8}
$$

Доказательство. Пусть $A$ является слабо $\beta_{3}$-ИС множеством с ЧРФ $f(x ; y)$. Если из трех чисел $x, y, z$ в точности одно принадлежит $A$, скажем $x$, то вычисляются $f(x, y)$ и $f(x, z)$ и оказываются равными $x$. Полагаем $g(x, y, z)=x$. В то же время

$$
(f(x, y)=f(x, z)=x \& x \notin A \Rightarrow y, z \notin A) \Rightarrow \beta_{8}(\bar{x}, \bar{y}, \bar{z})=0 .
$$

Если выяснится, что $f(y, x)=f(y, z)=y$ или $f(z, x)=f(z, y)=z$, то положим, соответственно $g(x, y, z)=y$ или $g(x, y, z)=z$. Иначе, считаем $g(x, y, z) \uparrow$. Ясно, что $A$ будет слабо $\beta_{8}$-ИС множеством с ЧРФ $g(x, y, z)$, то есть $K_{3} \subseteq K_{8}$. С другой стороны, $K_{8} \subseteq K_{3}$, так как $\beta_{8}(x, y, 0)=\beta_{3}(x, y)$, что и требовалось проверить.

Лемма 10. Справедливо включение

$$
K_{3} \subseteq K_{4}
$$

Доказательство. Пусть $A$ является слабо $\beta_{3}$-ИС множеством с ЧРФ $f(x, y)$. Если из трех чисел в точности два принадлежат $A$, скажем $x$ и $y$, то должны вычисляться $f(x, z)=z$ и $f(y, z)=y$. Полагаем $g(x, y, z)=x$. В то же время

$$
(f(x, z)=x \& f(y, z)=y \& x \notin A \Rightarrow z \notin A) \Rightarrow \beta_{4}(\bar{x}, \bar{y}, \bar{z})=0 .
$$

Если выяснится, что $f(x, y)=x$ и $f(z, y)=z$, то положим $g(x, y, z)=z$. Если же выяснится, что $f(y, x)=y$ и $f(z, x)=z$, то положим $g(x, y, z)=y$. Иначе, считаем $g(x, y, z) \uparrow$. Ясно, что $A$ будет слабо $\beta_{4}$-ИС множеством с ЧРФ $g(x, y, z)$, что и требовалось доказать.

Лемма 11. Справедливо равенство

$$
K_{3}=K_{6}
$$

Доказательство. Пусть $A$ является слабо $\beta_{3}$-ИС множеством с ЧРФ $f(x, y)$. Если $x=y$ или $x=z(y=z)$, то положим $g(x, y, z)=x$ (соответственно $y$ ). В противном случае, полагаем

$$
g(x, y, z)= \begin{cases}f(x, y), & \text { если первым вычислится значение } f(x, y) \\ f(x, z), & \text { если первым вычислится значение } f(x, z) \\ \uparrow, & \text { если } f(x, y) \uparrow \text { и } f(x, z) \uparrow\end{cases}
$$

Легко проверить, что $A$ оказывается слабо $\beta_{6}$-ИС множеством с ЧРФ $g$. Значит, $K_{3} \subseteq K_{6}$. Но $\beta_{6}(x, y, y)=\beta_{3}(x, y)$, что влечет включение $K_{6} \subseteq K_{3}$.

Лемма 12. Справедливо равенство

$$
K_{1}=K_{7}
$$


Доказательство. Пусть $A$ является слабо $\beta_{7}$-ИС множеством с ЧРФ $f(x, y, z)$. Вычисляем одновременно $f(x, y, z)$ и $f(x, z, y)$. Если оба значения неопределены, то и $g(x, y, z) \uparrow$. Пусть первым вычислилось $f(x, y, z)$. Положим

$$
g(x, y, z)= \begin{cases}f(x, z, y), & \text { если } f(x, y, z)=x, \\ f(y, z, x), & \text { если } f(x, y, z)=y \\ z, & \text { если } f(x, y, z)=z .\end{cases}
$$

Если же первым вычислилось $f(x, z, y)$, то пусть

$$
g(x, y, z)= \begin{cases}f(x, y, z), & \text { если } f(x, z, y)=x \\ y, & \text { если } f(x, z, y)=y \\ f(z, y, x), & \text { если } f(x, z, y)=z\end{cases}
$$

Разумеется, если в правой части значение неопределено, то и $g(x, y, z) \uparrow$.

Утверждаем, что $A$ оказывается слабо $\beta_{1}$-ИС множеством с ЧРФ $g(x, y, z)$. Это понятно, если $x, y, z \in A$. Иначе, пусть, например, $y \in A$ и $x, z \notin A$. Тогда $f(x, y, z) \downarrow$ и $f(x, y, z)=y$. Если $f(x, y, z)$ будет вычислено первым, то $g(x, y, z)=f(y, z, x)=y$. Если же первым будет вычислено значение $f(x, z, y)$ и оно не равно $y$, то

$$
f(x, y, z) \downarrow \& f(z, y, x) \downarrow \&(g(x, y, z)=f(x, y, z)=y=f(z, y, x)) .
$$

Остальные случаи рассматриваются аналогично. Значит, $K_{7} \subseteq K_{1}$. С другой стороны, $\beta_{7} \rightarrow \beta_{1}$ - тождественно истинная формула. По лемме $3 K_{1} \subseteq K_{7}$, что и доказывает данную лемму.

Следствие 2. Справедливъ включения

$$
K_{0} \subseteq K_{1} \subseteq K_{3} \subseteq K_{4} \subseteq K_{5}, \quad K_{0} \subseteq K_{2} \subseteq K_{4}
$$

Доказателъство. То, что $K_{0}$ и $K_{5}$ - наименьший и наибольший классы среди всех $K(\beta)$ следует из следствия 1 . В частности, $K_{0} \subseteq K_{1}$ и $K_{0} \subset K_{2}$. Так как $\beta_{1}(x, y, 0)=$ $\beta_{3}$, по лемме $2 K_{1} \subseteq K_{3}$. Но $\beta_{4} \rightarrow \beta_{2}$ - тождественно истинная БФ и по лемме 3 $K_{2} \subseteq K_{4}$. Наконец, по лємме $10 K_{3} \subseteq K_{4}$.

Результат следствия 2 изображен на диаграмме, приведенной на рис. 1 .

Возникает серия вопросов: являются ли включения, указанные в следствии 2, строгими. Мы ответим на все вопросы утвердительно, за исключением включения $K_{0} \subset K_{1}$. Если ответ на него также положителен, то остается открытым еще один вопрос: верно ли, что $K_{1} \subseteq K_{2}$ ?

Предложение 1. Существует множество $B$, не являющееся рекурсивно-перечислимым, и общерекурсивная функция $f$ такие, что

$$
(\forall x, y, z)(|B \cap\{x, y, z\}| \geqslant 2 \Rightarrow f(x, y, z) \in B) .
$$

Кроме того, $B={ }_{m} \mathbf{N} \backslash B$.

Доказательство. Пусть $A$ - нерекурсивное полурекурсивное множество [2],

$$
B=\{2 x: x \in A\} \cup\{2 x+1: x \notin A\} .
$$




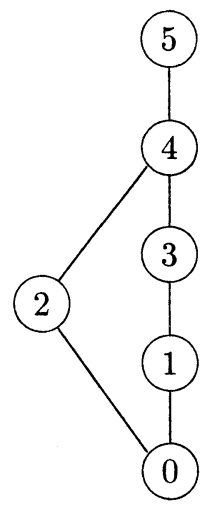

Рис. 1.

Ясно, что $B$ - не рекурсивно-перечислимое множество и $B={ }_{m} \mathbf{N} \backslash B$, так как $2 x \in B \Longleftrightarrow 2 x+1 \in \mathbf{N} \backslash B$. Так как $A$ полурекурсивное, существует селекторная общерекурсивная функция $g$ такая, что

$$
(\forall x, y)(x \in A \vee y \in A \Longleftrightarrow g(x, y) \in A) .
$$

Пусть также $g^{\prime}(x, y)=x$, если $g(x, y)=y$, и наоборот. Возьмем произвольные три числа. Если не менее двух из них равные, то положим $f(x, y, z)$, равной одному из этих равных чисел. Если же они разные, то не менее двух из них имеют одну и ту же четность. Предположим, что они суть $2 u$ и $2 v$. Положим $f(x, y, z)=2 g(u, v)$. Если же они имеют вид $2 u+1$ и $2 v+1$, то положим $f(x, y, z)=2 g^{\prime}(u, v)+1$. Нетрудно понять из определения $B$ и свойств функции $g$, что $f$ удовлетворяет условию предложения.

Следствие 3. Справедливы соотночения

$$
K_{2} \nsubseteq K_{3}, \quad K_{4} \nsubseteq K_{3} .
$$

Доказательство. Пусть $A, B$ и $f$ такие, как в предложении 1. Тогда $B$ будет как слабо $\beta_{2}$-ИС, так и слабо $\beta_{4}$-ИС множеством даже с общерекурсивной функцией $f$. Предположим, что $B$ является слабо $\beta_{3}$-ИС множеством с ЧРФ $h(x, y)$. Тогда $h(2 n, 2 n+1)$ всегда определено и

$$
n \in A \Longleftrightarrow h(2 n, 2 n+1)=2 n .
$$

Это противоречит нерекурсивности множества $A$.

Лемма 13. Дополнение любого рекурсивно-перечислимого множества А является как слабо $\beta_{3}-И С$, так и слабо $\beta_{4}-И С$ множеством.

Доказательство. Ясно, что $\bar{A}=\mathbf{N} \backslash A$ будет слабо $\beta_{4}$-ИС множеством со следующей ЧРФ $f$ : имея три числа $x, y$ и $z$ перечисляем $A$, пока одно из них, например, $x$, не вычислится в $A$. Полагаем $f(x, y, z)=y$ (или $z$ ). Если же $x, y, z \in \bar{A}$, то $f(x, y, z) \uparrow$. Аналогично показывается, что $\bar{A}$ будет и слабо $\beta_{3}$-ИС множеством. 
Предложение 2. Справедливо соотношение

$$
K_{3} \nsubseteq K_{1}
$$

Доказательство. Для каждого $n$ вычислим $\varphi_{n}(3 n, 3 n+1,3 n+2)$, где $\varphi_{n}$ - трехместная ЧРФ с клиниевским номером $n$. Если

$$
\varphi_{n}(3 n, 3 n+1,3 n+2) \in\{3 n, 3 n+1,3 n+2\}
$$

то относим к $A$ само значение $\varphi_{n}(3 n, 3 n+1,3 n+2)$. В противном случае числа $3 n, 3 n+1,3 n+2$ не принадлежат $A$.

Так как $A$ - рекурсивно-перечислимое множество, по лемме $13 \bar{A}$ является слабо $\beta_{3}$-ИС множеством. Предположим, что $\bar{A}$ слабо $\beta_{1}$-ИС множество с ЧРФ $f(x, y, z)$. Найдется номер $n$ такой, что $f(x, y, z)=\varphi_{n}(x, y, z)$. Рассмотрим значение $y=$ $\varphi_{n}(3 n, 3 n+1,3 n+2)$. Если оно не определено, то по построению $3 n, 3 n+1,3 n+2 \in \bar{A}$, a поэтому $\theta=\beta_{1}(\overline{3 n}, \overline{3 n+1}, \overline{3 n+2})=1$. Следовательно, значение $y$ определено и $y \in\{3 n, 3 n+1,3 n+2\}$. По построению в точности одно из трех $3 n, 3 n+1$ и $3 n+2$, именно $y$, принадлежит $A$. Но это означает, что, хотя $\theta=1$, но $y \in A$, что противоречит соответствию $f$ множеству $\bar{A}$.

Предложение 3. Справедливо соотношение

$$
K_{5} \nsubseteq K_{4}
$$

Доказательство. Для каждого $n$, если $y=\varphi_{n}(3 n, 3 n+1,3 n+2) \downarrow$ и справедливо включение $y \in\{3 n, 3 n+1,3 n+2\}$, то число $y$ относим к $A$. В противном случае к $A$ относим число $3 n$. Легко проверить, что $\bar{A} \notin K_{4}$, но $\bar{A} \in K_{5}$ по лемме 4 .

Следствие 4. Справедливы строгие включения

$$
K_{1} \subset K_{3} \subset K_{4} \subset K_{5}, \quad K_{0} \subset K_{2} \subset K_{4}
$$

причем $K_{2} \nsubseteq K_{3}$.

\section{Список литературы}

1. Дегтев А. Н., Импликативно селекторные множества. Алгебра и логика (1996) 35, №2, $145-153$.

2. Jockusch C. G., Owings J. C., Weakly semirecursive sets. J. Symb. Logic (1990) 55, №2, 637-644.

3. Kummer M., Stephan F., Weakly semirecursive sets and r. e. orderings. Ann. Pure and Appl. Logic (1993) 60, 133-150. 